\title{
Validação da \\ Metodologia Analítica de Granulometria para a Penincilina G Benzatina
}

\section{Rodrigo C. de Oliveira \& Marcell Gamboa}

A Penicilina G Benzatina é um antibiótico que faz parte do grupo dos $\beta$-lactâmicos, com eficácia no tratamento de sífilis e na profilaxia da febre reumática. $\mathrm{O}$ interesse principal deste trabalho é propor a validação do método granulométrico desta substância por meio da técnica de espalhamento de luz (Difração a Lazer), onde, as partículas ficam dispersas num fluido em movimento sobre a qualé incidido um feixe de luz monocromático (Laser). Os resultados obtidos na validação foram tratados estatisticamente por análise de desvio padrão. $\mathrm{O}$ método demonstrou-se atender aos requisitos de boas práticas em laboratórios (BPL) exigidos pela legislação brasileira em vigor, RE n 899 de 29 de maio de 2003 da Agência Nacional de Vigilância Sanitária, sendo, portanto, preciso e confiável para a medição das partículas da Penicilina G Benzatina.

Palavras-chave: tamanho de partícula, espalhamento de luz, validação.

Is the Penicilina $\mathrm{G}$ Benzatina an antibiotic that is part of the group of the $\beta$-lactâmicos, with effectiveness in the syphilis treatment and in the prophylaxis of the rheumatic fever. The main interest of this work is to propose the validation of the method particle size of this substance through the technique of light scattering (Laser Diffraction), where, the particles are dispersed in a fluid in movement on which a monochrome light bunch is happened (Laser). The results obtained in the validation statistics were treated by analysis of standard deviation. The method demonstrated to assist to the requirements of good practices in laboratories (BPL) demanded by the Brazilian legislation in vigor, RE $n^{\circ} 899$ of May 29, 2003 of the National Agency of Sanitary Surveillance, being, therefore, need and reliable for the measurement of the particles of the Penicilina $G$ Benzatina.

Keywords: particle size, light scattering, validation. 


\section{Introdução}

$\mathrm{O}$ presente artigo foi concebido sob o signo da objetividade e da simplicidade sem, contudo, abrir mão do rigor da informação e da abrangência do conteúdo sobre validação do método de tamanho de partículas para a Penicilina G Benzatina.

A tecnologia do estudo do tamanho de partículas tem desempenhado um papel de importância crescente na tecnologia moderna. Isto se deve tanto ao seu potencial de conservação de energia e de materiais, quanto à sua capacidade de produzir produtos que, de outro modo, seriam processados a um custo maior e ou com qualidade inferior.

$\mathrm{Na}$ indústria farmacêutica a determinação do tamanho de partículas de matérias-primas é de grande importância, pois por meio dos resultados desta técnica pode-se desenvolver e produzir medicamentos, em que sua biodisponibilidade, desintegração e dissolução não serão afetadas.

Para o futuro, as expectativas são de que os produtos gerados a partir do estudo do tamanho de suas partículas exerçam uma função preponderante em termos globais como produtos de alto desempenho para aplicações críticas envolvendo tecnologia avançada e desta maneira contribuindo para que a ciência e a tecnologia cumpram o seu papel de prover a melhoria do bem estar da humanidade.

Foi utilizado o equipamento da Malvern denominado Mastersizer 2000 para determinar o tamanho das partículas da Penicilina G Benzatina. Este equipamento é baseado no Método de Espalhamento de Luz Lazer e Difração Fraunhofer. O princípio de operação deste equipamento é fazer com que as partículas fiquem dispersas num fluido em movimento sobre a qual é incidido um feixe de luz monocromático (Laser). Ao atingir uma quantidade de partículas, a luz incidente sofre uma interação segundo quatro diferentes fenômenos (difração, refração, reflexão e absorção) formando um invóculo tridimensional de luz. $\mathrm{O}$ formato e o tamanho deste invóculo são afe tados pelo índice de refração relativo da partícula no meio dispersante, pelo comprimento de onda da luz e pelo tamanho e formato das partículas. Detectores estrategicamente espalhados medem a intensidade e o ângulo da luz espalhada. O sinal é então convertido para distribuição de tamanho de partículas através de algoritmos matemáticos que podem ser a teoria de espalhamento Mie ou pela teoria de difração de Fraunhofer ${ }^{1,2,3,4}$.

A teoria de espalhamento Mie apresenta uma solução matemática para o espalhamento de luz incidente sobre partículas esféricas e pode ser aplicada para partículas com diferentes formatos e razões de aspecto. Para sua aplicação, porem, é necessário um conhecimento prévio dos índices de refração do material que está sendo analisado e do meio em que ele se encontra ${ }^{2,3,4}$.

A teoria de difração de Fraunhofer é aplicada nos casos onde as partículas são opacas e maiores que o comprimento de onda da luz. A partícula altera a intensidade e o ângulo do feixe de luz espalhado, com utilização do laser, o ângulo de espalhamento é inversamente proporcional ao diâmetro da partícula (assume-se a forma esférica) ${ }^{2,3,4}$.

A análise granulométrica pode ser feita utilizando a via seca onde o meio dispersante é o ar. Esta técnica tem como princípio a dispersão da amostra em ar, através da aceleração das partículas a velocidades próximas à do som $(300 \mathrm{~m} / \mathrm{s})$, na esperança de que as forças de cisalhamento irão separar e dispersar as partículas agregadas ${ }^{5,6}$.

Método utilizado principalmente para partículas inorgânicas, pois a pressão utilizada pode causar fragmentações nas partículas orgânicas maiores que $25 \mu \mathrm{m}$ e aglomeração nas partículas menores que $5 \mu \mathrm{m}^{5,6}$.

A determinação granulométrica por via úmida tem como princípio a dispersão da amostra em um líquido. Esta técnica é muito utilizada para partículas orgânicas e partículas menores que $25 \mu \mathrm{m}$ pelo fato de maior dispersão destas partículas em meio líquido.

A principal dificuldade na utilização desta técnica é a escolha do líquido correto para conseguir uma dispersão estável, pois a amostra não pode dissolver e nem formar aglomerados durante o processo, se necessário utilizar surfactante para diminuir a tensão superficial do meio dispersante. A utilização do ultra-som durante o processo pode diminuir a presença de aglomerados, mas em excesso pode causar a fragmentação das partículas.

Têm-se então, como objetivo a validação do método granulométrico de Penicilina G Benzatina e provar por meio dos parâmetros de repetibilidade, precisão e precisão intermediária que o método utilizado é eficaz e 
seguro, tornando os resultados mais próximo possíveis do valor real.

O método de tamanho de partículas por não constar em nenhum compêndio oficial tem que ser validado e a análise de tamanho de partícula é um dos fatores físicoquímicos que pode afetar a biodisponibilidade de um fármaco. Portanto, diante da importância do assunto para a tecnologia farmacêutica nacional, espera-se dar uma importante contribuição para a melhoria do senso crítico de novos e antigos usuários, bem como de operadores dos equipamentos envolvidos, em buscar desenvolver e validar novas metodologias confiáveis de tamanho de partículas para outras substâncias ${ }^{3,4,7}$.

\section{Metodologia}

\section{Procedimento}

Foi transferido $100 \mathrm{mg}$ de Penicilina G Benzatina para o medidor de partículas marca Malvern Mastersizer modelo Hydro 2000S (A). Para atingir a obscuração desejável $(3 \%$ a $30 \%)$ a amostra ficou 5 minutos no medidor de partículas a uma rotação de $1855 \mathrm{rpm}$ e sobre tratamento de ultrasom (5\%). Após esse tempo foi iniciado as medidas do tamanho das partículas, sendo que os parâmetros utilizados foram:

- Fluido: Água destilada

- Índice de refração: 1,45 - 0,001

- Tempo de Medida: 6 segundos

- Tempo de Background: 6 segundos

A medição das partículas da amostra é devido à interação do feixe de luz (fonte de $\mathrm{He}-\mathrm{Ne}$ de baixa potência $2 \mathrm{~mW}$ ) com as partículas da Penicilina G Benzatina em água destilada. Quando este feixe de luz atinge uma quantidade de partículas da amostra ocorre o espalhamento de luz, sendo que uma parte da luz será absorvida e a outra transmitida. O espalhamento inclui a luz refratada, refletida e difratada. Desta forma, o diâmetro médio das partículas da Penicilina $\mathrm{G}$ Benzatina é, então, medido pela intensidade de energia da luz em determinados ângulos de espalhamento. Um conjunto de lentes, detectores fotos-elétricos contidos no equipamento e um microprocessador captão a intensidade de energia espalhada e, assim, tem-se a distribuição granulométrica de partículas.

\section{Estudo de Validação}

A precisão foi avaliada com relação à reprodutibilidade e a precisão intermediária. A reprodutibilidade foi determinada pela análise em sextuplicada da mesma amostra. A precisão intermediária foi determinada em dias diferentes por analistas diferentes.

\section{Resultados e Discussão}

O comparativo entre o fluído utilizado (Água destilada) e a amostra (Penicilina G Benzatina) está demonstrado no gráfico de Background (Figura 1):

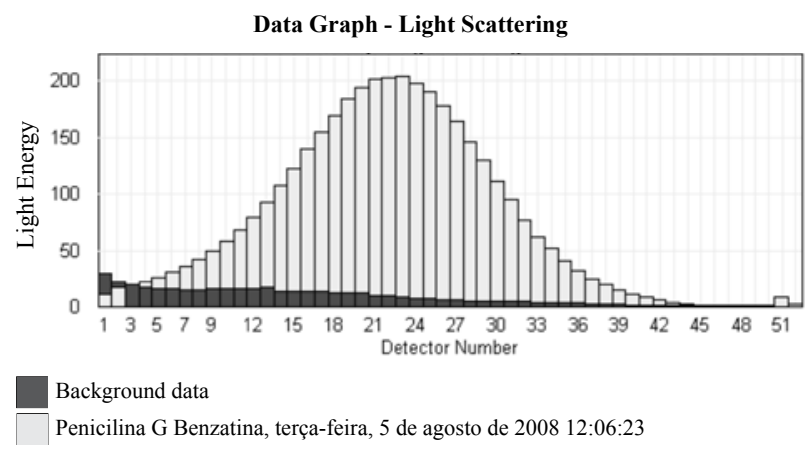

Figura 1 - Gráfico de Background

Os resultados obtidos na repetibilidade, precisão e precisão intermediária estão resumidos nas Tabelas 1, 2, 3 que correspondem aos histogramas 1 (Figura 2), 2 (Figura 3) e 3 (Figura 4).

\section{Repetibilidade}

Tabela 1 - Resumo dos resultados da análise de repetibilidade.

\begin{tabular}{|c|c|c|c}
\hline \multirow{2}{*}{ Testes } & $\mathbf{D}(\mathbf{0 . 1 0})$ & $\mathbf{D}(\mathbf{0 . 5 0})$ & $\mathbf{D}(\mathbf{0 . 9 0})$ \\
\cline { 2 - 4 } & $\boldsymbol{\mu m}$ & $\boldsymbol{\mu m}$ & $\boldsymbol{\mu m}$ \\
\hline $\mathbf{1}$ & 5,73 & 15,16 & 38,25 \\
\hline $\mathbf{2}$ & 5,79 & 15,02 & 37,4 \\
\hline $\mathbf{3}$ & 5,76 & 15,20 & 38,7 \\
\hline $\mathbf{4}$ & 5,78 & 15,25 & 38,12 \\
\hline $\mathbf{5}$ & 5,73 & 14,70 & 38,17 \\
\hline $\mathbf{6}$ & 5,75 & 14,90 & 38,13 \\
\hline Média & $\mathbf{5 , 7 6}$ & $\mathbf{1 5 , 0 4}$ & $\mathbf{3 8 , 1 3}$ \\
\hline SD & $\mathbf{0 , 0 2 5}$ & $\mathbf{0 , 2 1 0}$ & $\mathbf{0 , 4 1 8}$ \\
\hline RSD (\%) & $\mathbf{0 , 4 3 5}$ & $\mathbf{1 , 3 9 3}$ & $\mathbf{1 , 0 9 6}$ \\
\hline
\end{tabular}




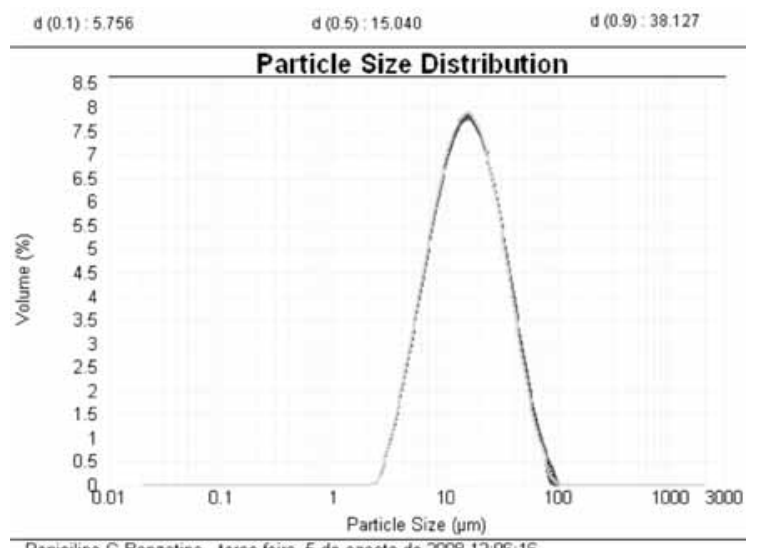

-Penicilina G Benzatina, terça-feira, 5 de agosto de $200812: 06: 16$

- Penicilina G Benzatina , terça-feira, 5 de agosto de $200812: 06: 23$

-Penicilina G Benzatina, terça-feira, 5 de agosto de $200812.06: 36$

-Penicilina G Benzatina, terça-feira, 5 de agosto de $200012: 06: 42$

-Penicilina G Benzatina, terça-feira, 5 de agosto de 2008 12:06:49

Penicilina G Benzatina , terça-feira, 5 de agosto de 200812.06 .56

Figura 2 - Histograma de sobreposição do teste de repetibilidade realizado em sextuplicata.

Tabela 2 - Resumo dos resultados da análise de precisão.

\begin{tabular}{|c|c|c|c}
\hline \multirow{2}{*}{ Testes } & $\mathbf{D}(\mathbf{0 . 1 0})$ & $\mathbf{D}(\mathbf{0 . 5 0 )}$ & $\mathbf{D}(\mathbf{0 . 9 0})$ \\
\cline { 2 - 4 } & $\boldsymbol{\mu m}$ & $\boldsymbol{\mu m}$ & $\boldsymbol{\mu m}$ \\
\hline $\mathbf{1}$ & 5,86 & 15,6 & 37,54 \\
\hline $\mathbf{2}$ & 5,85 & 15,21 & 37,15 \\
\hline $\mathbf{3}$ & 5,87 & 15,28 & 37,78 \\
\hline $\mathbf{4}$ & 5,86 & 15,21 & 37,60 \\
\hline $\mathbf{5}$ & 5,88 & 15,19 & 37,10 \\
\hline $\mathbf{6}$ & 5,78 & 15,11 & 37,20 \\
\hline Média & $\mathbf{5 , 8 5}$ & $\mathbf{1 5 , 1 1}$ & $\mathbf{3 7 , 4 0}$ \\
\hline SD & $\mathbf{0 , 0 3 5}$ & $\mathbf{0 , 2 5 1}$ & $\mathbf{0 , 2 8 1}$ \\
\hline RSD (\%) & $\mathbf{0 , 6 1 1}$ & $\mathbf{1 , 6 6 2}$ & $\mathbf{0 , 7 5 3}$ \\
\hline \multicolumn{4}{|c}{}
\end{tabular}

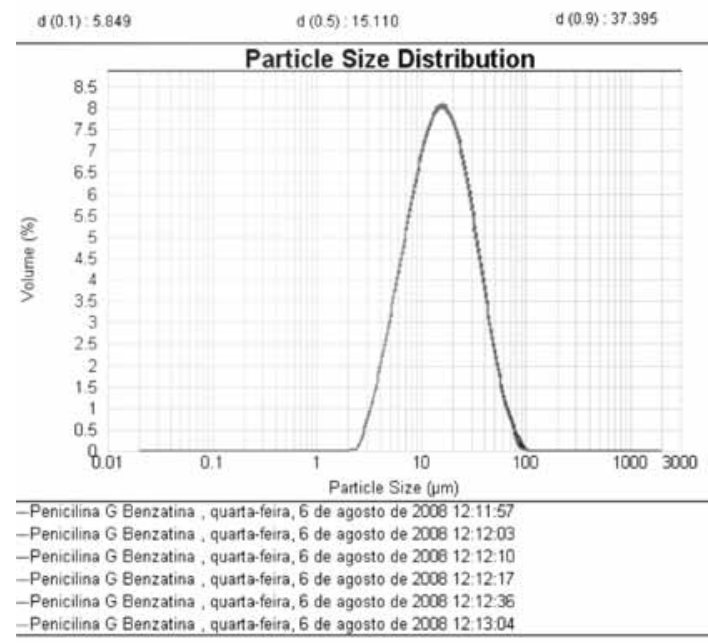

Figura 3 - Histograma de sobreposição da análise de precisão realizada em sextuplicata.

\section{Precisão Intermediária}

Tabela 3 - Resumo dos resultados da análise de precisão intermediária.

\begin{tabular}{|c|c|c|c|}
\hline \multirow{2}{*}{ Testes } & $\mathbf{D}(\mathbf{0 . 1 0})$ & $\mathbf{D}(\mathbf{0 . 5 0})$ & $\mathbf{D}(\mathbf{0 . 9 0})$ \\
\cline { 2 - 4 } & $\boldsymbol{\mu \mathrm { m }}$ & $\boldsymbol{\mu m}$ & $\boldsymbol{\mu m}$ \\
\hline $\mathbf{1}$ & 5,36 & 14,33 & 36,6 \\
\hline $\mathbf{2}$ & 5,42 & 14,41 & 37,38 \\
\hline $\mathbf{3}$ & 5,41 & 14,39 & 37,16 \\
\hline $\mathbf{4}$ & 5,42 & 14,37 & 37,17 \\
\hline $\mathbf{5}$ & 5,41 & 14,32 & 36,96 \\
\hline $\mathbf{6}$ & 5,4 & 14 & 37,05 \\
\hline Média & $\mathbf{5 , 4}$ & $\mathbf{1 4 , 3}$ & $\mathbf{3 7 , 0 5}$ \\
\hline SD & $\mathbf{0 , 0 2 3}$ & $\mathbf{0 , 1 5 3}$ & $\mathbf{0 , 2 6 3}$ \\
\hline RSD (\%) & $\mathbf{0 , 4 1 7}$ & $\mathbf{1 , 0 6 6}$ & $\mathbf{0 , 7 1 0}$ \\
\hline
\end{tabular}

\section{Repetibilidade}

Os resultados obtidos (Tabela 1) representam a eficácia do equipamento e do método em relação a repetição dos resultados para seis análises da mesma amostra de penicilina $\mathrm{G}$ benzatina.

Adotou-se o seguinte critério de aceitação para este teste de repetibilidade:

O desvio padrão relativo RSD \% para as seis leituras deve apresentar valores $<2 \%$ para os diâmetros (D0.10, D0.50 e D0.90).

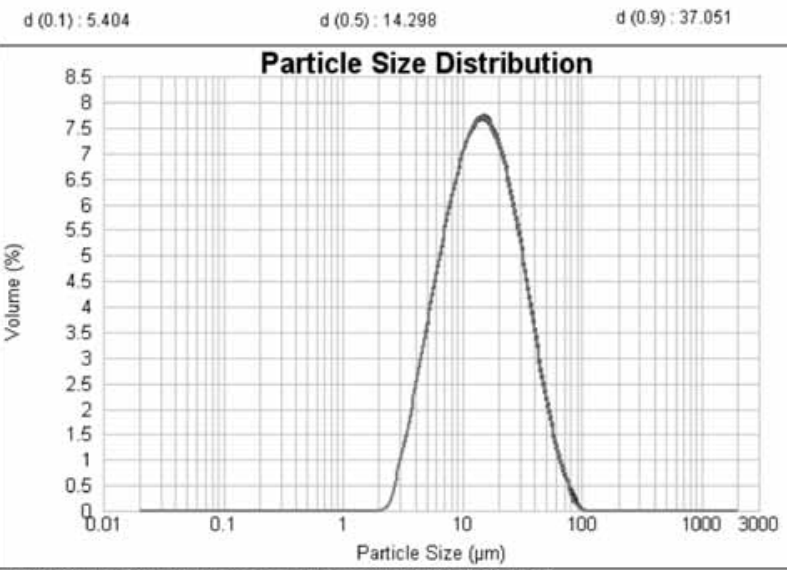

-Penicilina G Benzatina, domingo, 10 de agosto de 2008 14:11:27

-Penicilina G Benzatina, domingo, 10 de agosto de 2008 14:11:34

-Penicilina G Benzatina, domingo, 10 de agosto de 2008 14:11:40

-Penicilina G Benzatina, domingo, 10 de agosto de 2008 14:11:47

-Penicilina G Benzatina, domingo, 10 de agosto de 2008 14:12:00

-Penicilina G Benzatina, domingo, 10 de agosto de 2008 14:12:20

Figura 4 - Histograma de sobreposição da análise de precisão intermediária realizada em sextuplicata. 


\section{Precisão}

Os resultados obtidos (Tabela 2) no teste de precisão é a avaliação da reprodutibilidade dos valores dos diâmetros (D0.10, D0.50 e D0.90) obtidos em 6 (seis) medidas de uma amostragem múltipla de uma mesma amostra da Penicilina G Benzatina.

Adotou-se o seguinte critério de aceitação para este teste de precisão:

O desvio padrão relativo RSD \% para as seis leituras deve apresentar valores para $\mathrm{D}(0.50)<3 \%$ e $\mathrm{D}(0.10)$ e $\mathrm{D}(0.90)<5 \%$ para produtos que apresenta tamanho médio $\mathrm{D}(0.50)>10 \mu \mathrm{m}$. Para produtos com $\mathrm{D}(0.50)<10$ $\mu \mathrm{m}$, uma boa reprodutibilidade é aquela em que, o RSD $\%$ de $\mathrm{D}(0.50)<6 \%$ e $\mathrm{D}(0.90)$ e $\mathrm{D}(0.10)<10 \%$.

Observando-se os resultados das tabelas 2 e 3, fica evidente que o método atende o critério de aceitação $(\mathrm{D}(0.50)<3 \%$ e $\mathrm{D}(0.10)$ e $\mathrm{D}(0.90)<5 \%)$, pois o tamanho médio das partículas para $\mathrm{D}(0.50)$ apresentou-se maior que $10 \mu \mathrm{m}$ (dez micrômetros).

\section{Precisão Intermediária}

A checagem da precisão intermediária do método é feita por um segundo analista em dia diferente.

Os resultados dos dois analistas devem ser combinados para que a média do RSD \% D(0.50) seja < que 3\%.

Na Tabela 2 e 3, pode-se observar a comparação entre os resultados obtidos pelo $1^{\circ}$ e $2^{\circ}$ analista. O RSD $\% \mathrm{D}(0.50)$ encontrado pelo $1^{\circ}$ analista foi de $1,662 \%$ e para o $2^{\circ}$ analista foi de $1,066 \%$. Portanto, a média do desvio padrão relativo RSD \% $\mathrm{D}(0,50)$ é igual 1,364 $\%$, atendendo o critério de aceitação estabelecido para a precisão intermediária.

\section{Conclusão}

Os resultados da validação obtidos neste estudo demonstraram que o método de tamanho de partícula para a Penicilina G Benzatina é satisfatório para seu uso planejado, atendendo os requisitos de boas práticas de laboratório ${ }^{8,9}$.

Do exposto, pode-se concluir que, de acordo com o princípio físico no qual cada método de análise de tamanhos de partículas seja desenvolvido utilizando o medidor de partículas da MALVERN, pode-se chegar a resultados eficazes e seguro, porque:

- A medida é não intrusiva, isto é, a medida ocorre dentro do aparelho, sem a interferência do meio ou do operador;

- A repetibilidade é de $+/-2 \%$;

- O instrumento é muito fácil de ajustar e operar.

Estudar e propor modelos matemáticos que permitam correlacionar a validação entre técnicas de partículas diferentes.

\section{BIBLIOGRAFIA}

1. Guardani, R.; Nascimento, C. A. O.; Onimaru, R. S. 2002, 126, 42. ANVISA, Agência Nacional de Vigilância Sanitária. Resolução n.899, 2003.

2. Hausner, H.H.; Powder Technol. 1981, $30,3$.

3. Malvern Instruments, Sample Dispersion \& Refractive Index Guide, Malvern Instruments Ltd.: Spring Lane South, 1996.

4. Rawle, A.; Basic Particles of Particle Size Analysis. Disponível em: $<$ http://www.malvern.co.uk $>$. Acesso em: 12/05/2008.

5. Iacocca, R. G.; ASM Handbook, 1998, 7, 234.

6. Jillavenkatesa, A.; Dapkunas S. J.; Lum L. H. Goetzel, G. C. NIST Recommended Practice guide - Special Publication 960 - 1, Washington, 2001.

7. Vogel, A. I.; Análise Química Quantitativa, 4 ed., Rio de Janeiro: Guanabara Koogan, 1992.

8. ANVISA, Resolução - RDC n. 210. Disponível em: $<\mathrm{http}$ ://www.anvisa.gov.br>. Acesso em: 14/05/2008.

9. ANVISA, Agência Nacional de Vigilância Sanitária. Resolução n. 899, 2003.

\section{Rodrigo C. de Oliveira' \& Marcell Gambôa*2}

\footnotetext{
${ }^{1}$ IPOG - Faculdades Oswaldo Cruz, Goiânia, GO, Brasil

${ }^{2}$ Faculdade de Tecnologia SENAI Roberto Mange, Anápolis, GO, Brasil

*E-mail: marcellgamboa@hotmail.com
} 